AUS DER FAKULTÄT FÜR MEDIZIN

DER UNIVERSITÄT REGENSBURG

PD DR. DR. CHRISTOPH KLINGELHÖFFER

MUND-, KIEFER- UND GESICHTSCHIRURGIE

\title{
SURGICAL TREATMENT OF ADVANCED MEDICATION-RELATED OSTEONECROSIS OF THE JAWS: COMPARISON OF SOFT TISSUE CLOSURE TECHNIQUES AND EVALUATION OF SIDE EFFECTS
}

\author{
Inaugural - Dissertation \\ zur Erlangung des Doktorgrades \\ der Medizin \\ der \\ Fakultät für Medizin \\ der Universität Regensburg
}

vorgelegt von

Greta Lou Ahrenbog 

AUS DER FAKULTÄT FÜR MEDIZIN

DER UNIVERSITÄT REGENSBURG

PD DR. DR. CHRISTOPH KLINGELHÖFFER

MUND-, KIEFER- UND GESICHTSCHIRURGIE

\title{
SURGICAL TREATMENT OF ADVANCED MEDICATION-RELATED OSTEONECROSIS OF THE JAWS: COMPARISON OF SOFT TISSUE CLOSURE TECHNIQUES AND EVALUATION OF SIDE EFFECTS
}

\author{
Inaugural - Dissertation \\ zur Erlangung des Doktorgrades \\ der Medizin \\ der \\ Fakultät für Medizin \\ der Universität Regensburg
}

vorgelegt von

Greta Lou Ahrenbog 
Dekan:

1. Berichterstatter:

2. Berichterstatter:

Tag der mündlichen Prüfung:
Prof. Dr. Dirk Hellwig

PD Dr. Dr. Christoph Klingelhöffer

Prof. Dr. Dr. Peter Proff

07.06.2021 
Gemäß der Promotionsordnung der medizinischen Fakultät der Universität Regensburg ( 6 Absatz 7) wird eine wissenschaftliche Originalarbeit, welche unter Erstautorenschaft veröffentlicht wurde, eingereicht. Dieser ist eine erläuternde Zusammenfassung vorangestellt. 


\section{Inhalt}

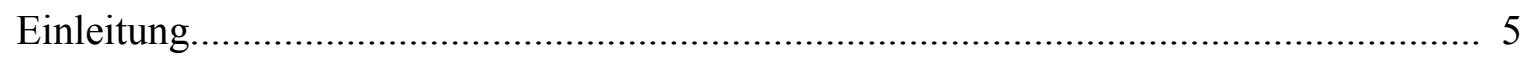

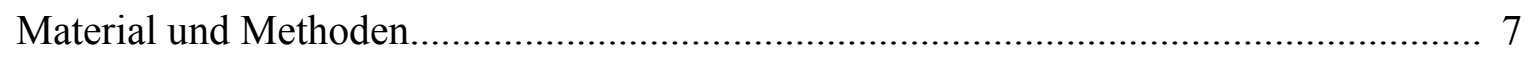

Patientenmerkmale und Datenerhebung............................................................ 7

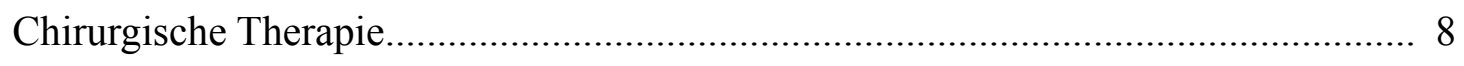

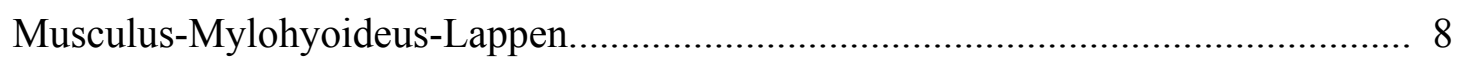

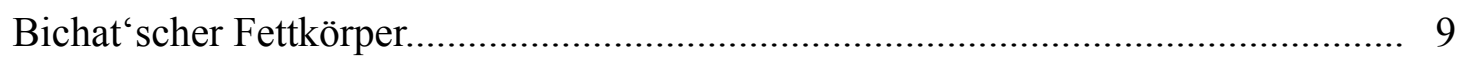

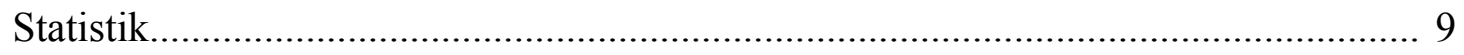

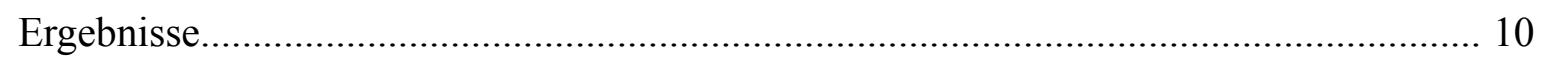

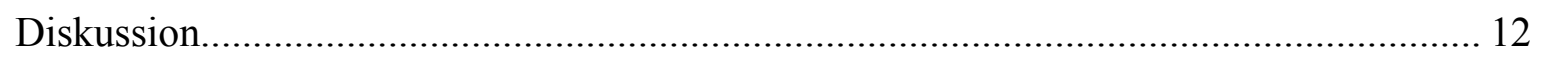

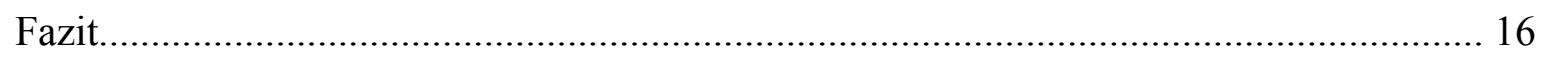

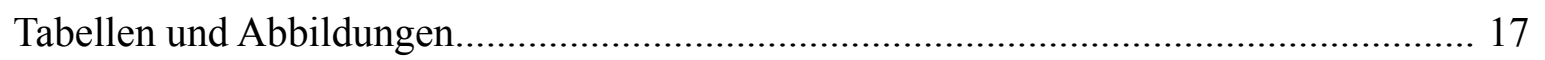

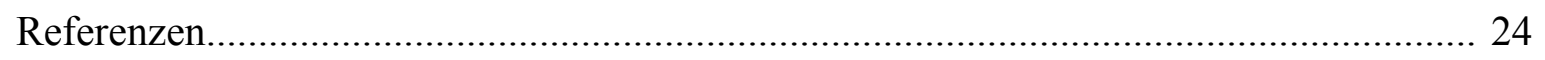

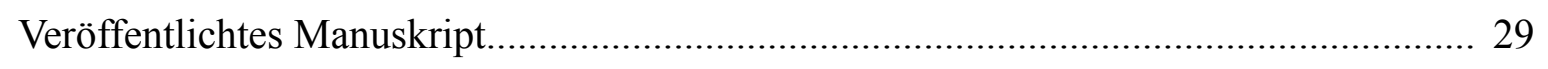

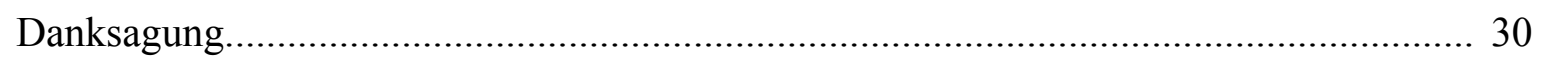




\section{EINLEITUNG}

Eine Medikamenten-assoziierte Kiefernekrose (MRONJ, medication-related osteonecrosis of the jaw) ist eine unerwünschte Wirkung von antiresorptiven Medikamenten wie Bisphosphonaten oder Denosumab (Marx, 2003; Amgen Canada Inc., 2019; Lee et al., 2014). Diese Medikamente inhibieren Osteoklasten und dadurch die Knochenresorption (Amgen Canada Inc., 2019; Russell et al., 2008). Die Pathogenese der Kiefernekrosen ist noch nicht vollständig verstanden.

Die häufigsten Indikationen für eine antiresorptive Therapie sind knochenkonsumierende maligne Erkrankungen und Knochenmetastasen, aber auch benigne Erkrankungen wie Osteoporose (Otto et al., 2012; Saussez et al., 2009). Die Prävalenz von MRONJ schwankt zwischen $0.0043 \%$ und $5.16 \%$ und scheint von Präparat, Dosis und der zugrundeliegenden Erkrankung abzuhängen (Rugani et al., 2016; Coleman et al., 2011; Hallmer et al., 2018; Lo et al., 2010; Mauri et al., 2009; Qi et al., 2014). Lokale Faktoren wie schlechte Mundhygiene oder Zahnextraktionen scheinen das Risiko für eine Kiefernekrose zu erhöhen (Hoff et al., 2008; Vahtsevanos et al., 2009). Präventive Maßnahmen sind somit ein wichtiger Baustein zur Verhinderung bzw. Früherkennung einer MRONJ (Marx et al., 2005; Hoff et al., 2008).

Seit Kiefernekrosen im Jahr 2003 erstmals als unerwünschte Wirkung der antiresorptiven Therapie beschrieben wurden, besteht eine Diskussion über die bestmögliche Stadien-basierte Behandlung (Ristow et al., 2015; Bodem et al., 2016; Carlson, 2014; Marx, 2003). Für das Initialstadium von MRONJ empfiehlt die American Association of Oral and Maxillofacial Surgery (AAOMS) ein konservatives Vorgehen basierend auf regelmäßigen Kontrollen, antibakteriellen Mundspülungen, Analgetika, einer oralen antibiotischen Therapie und/oder lokalem Débridement im Falle einer Infektion (Ruggiero et al., 2014). Eine chirurgische Therapie als Standardtherapie wird erst für Patienten mit fortgeschrittenen Nekrosen empfohlen. In diesem Stadium treten definitionsgemäß unter anderem extraorale Fisteln, oroantrale oder oronasale Verbindungen, ausgedehnte Osteolysen oder pathologische Frakturen auf (Ruggiero et al., 2014). 
Die etablierte chirurgische Behandlung der MRONJ besteht grundsätzlich aus (1) einer marginalen, segmentalen oder kontinuierlichen Resektion von nekrotischem und infizierten Knochen bis zur Darstellung einer suffizienten Durchblutung des Knochens, (2) dem Glätten von scharfen Knochenrändern und (3) der Bildung eines Mukoperiostlappens für einen spannungsfreien Wundverschluss. Die Spannbreite an positivem Outcome im Sinne einer intakten postoperativen Schleimhaut zu diesem Operationsverfahren ist hoch: $27.6 \%$ (Klingelhöffer et al., 2016); 43.8 \% (Reich et al., 2015); $51.1 \%$ (Bodem et al., 2016); 68 \% (Graziani et al., 2012); 88 \% (Wilde et al., 2011); $91.6 \%$ (Carlson and Basile, 2009).

Einige Studien haben bereits gezeigt, dass ein modifizierter Wundverschluss zu einem besseren chirurgischen Outcome führen kann (Mücke et al., 2016; Ristow et al., 2018; Wutzl et al., 2008).

Das Ziel dieser Studie war es, durch Einsatz des Musculus mylohyoideus bzw. des Bichat'schen Fettkörpers eine zusätzliche Defektdeckung zu schaffen und das Outcome dieses Verfahrens mit dem der alleinigen Defektdeckung durch einen Mukoperiostlappen zu vergleichen. Außerdem wurde versucht mögliche Einflussfaktoren und postoperative Risiken zu detektieren. Zusätzlich wurden einige Aspekte zur Lebensqualität der Patienten evaluiert. 


\section{MATERIAL UND METHODEN}

\section{Patientenmerkmale und Datenerhebung}

Diese Studie wurde durch die lokale Ethikkommission bewilligt (Nr. 16-101-0257). Zwischen September 2016 und März 2019 wurde die prospektive monozentrische klinische Studie durch die Klinik für Mund-, Kiefer- und Gesichtschirurgie des Universitätsklinikums Regensburg durchgeführt. Basierend auf der Klassifikation der American Association of Oral and Maxillofacial Surgeons (AAOMS) von 2009 und 2014 wurden 44 Fälle von MRONJ im Stadium III in unsere Studie eingeschlossen (Ruggiero et al., 2009; Ruggiero et al., 2014). Die Einschlusskriterien umfassten (1) das Vorhandensein einer MRONJ Stadium III laut Klassifikation der AAOMS, (2) eine chirurgische Intervention und (3) eine Nachsorgeperiode von mindestens 6 Monaten. Die folgenden Kriterien führten zum Ausschluss: (a) eine Bestrahlung von Kopf- oder Halsregion in der Anamnese, (b) Metastasen in den Kieferknochen, (c) eine Therapie, die von der im Verlauf beschriebenen Therapien abweicht.

Präoperativ wurden folgende Parameter gesammelt. Durch eine klinische Untersuchung wurde die genaue Lokalisation der MRONJ bestimmt. Es wurde sowohl zwischen Ober- und Unterkiefer als auch zwischen einem vorderen Bereich (zwischen den Dentes canini) und einem hinteren Bereich (Eckzahnregion bis zur Retromolarregion) unterschieden. Außerdem wurde der größte Durchmesser des freiliegenden Knochens ausgemessen. Im Orthopantomogramm wurde die Tiefenausdehnung über den Abstand von Knochenoberfläche zum tiefsten Punkt der Osteolyse bestimmt.

Einige Parameter wurden sowohl präoperativ als auch während der Nachsorge erfragt. Die Patienten ordneten ihr Schmerzlevel anhand einer Skala von 0 (kein Schmerz) bis 10 (größter vorstellbarer Schmerz) ein. Zudem wurde nach dem Vorhandensein von Halitosis gefragt. Die

prothetische Versorgung wurde evaluiert und als ,definitiv versorgt ${ }^{\star}$ angesehen, wenn die Patienten entweder Zahnprothesen verwendeten oder alle Stützzonen des Gebisses erhalten waren. Lippen und Zunge wurden klinisch auf Sensibilitätsstörungen getestet. Eine herabgesetzte Empfindung im Vergleich zur Gegenseite wurde als Taubheit gewertet. 
Als primärer Endpunkt dieser Studie wurde das Vorliegen einer intakten Schleimhaut ohne das Auftreten eines Rezidivs und ohne das Vorhandensein von Entzündungszeichen definiert. Entzündungszeichen umfassten Schwellungen, Fisteln, Pus und eine gerötete Schleimhaut. Ein Rezidiv entsprach dem Wiederauftreten von freiliegendem Knochen im Operationsbereich.

\section{Chirurgische Therapie}

Alle Patienten durchliefen einen 10-tägigen stationären Aufenthalt. In Allgemeinanästhesie wurde der nekrotische Knochen mit einem schmalen Rand von erkennbar vitalem Knochengewebe durch eine Knochensäge und durch Piezochirurgie entfernt. Anschließend wurden scharfe Knochenkanten und -oberflächen abgeschliffen bis eine ausreichende Durchblutung des Knochengewebes sichtbar war. Der Wundverschluss erfolgte durch verschiedene im Verlauf beschriebene Techniken. Die Patienten erhielten während des Aufenthalts eine perioperative intravenöse antibiotische Therapie mit Amoxicillin und Clavulansäure. Falls eine Allergie gegen Penicillin bekannt war, erhielten die Patienten Clindamycin. Außerdem wurde dreimal am Tag die antiseptische Mundspülung Chlorhexidin (CHX) $(0,12 \%)$ angewendet. Die Patienten wurden postoperativ über eine Magensonde ernährt. Die Nähte wurden am 14. postoperativen Tag entfernt.

Es wurde eine standardisierte Nachsorgeperiode in unserer Poliklinik festgelegt: 14 Tage postoperativ; 6 Wochen postoperativ; alle 3 Monate während des ersten Jahres nach der Operation, dann alle 6 Monate. Der kleinste Nachsorgezeitraum lag bei 6 Monaten, die durchschnittliche Nachsorgezeit umfasste 13,2 Monate. Zusätzlich wurde 6 Monate nach der Operation erneut ein Orthopantomogramm aufgenommen.

\section{Musculus-Mylohyoideus-Lappen}

Der Musculus mylohyoideus wurde nach der Resektion einer Unterkiefernekrose zur zusätzlichen Defektdeckung verwendet. Zunächst wurde der Muskel hierzu von der Linea mylohyoidea freipräpariert, von der Mukosa und dem Periost abgetrennt und über die verbliebene Mandibula gezogen. Währenddessen wurde darauf geachtet, den Nervus lingualis 
und den Ductus submandibularis nicht zu verletzen. Im Anschluss wurde der Muskel spannungsfrei am vestibulären Periost fixiert (Ristow et al., 2018; Lemound et al., 2012). Ein zusätzlicher Mukoperiostlappen ergänzte den vollständigen Wundverschluss. (Abbildung 1). Falls der Musculus mylohyoideus zu dünn für die Präparation oder der Defekt ungünstig lokalisiert war (anteriorer Kieferbereich), wurde nur der Mukoperiostlappen verwendet.

\section{Bichat'scher Fettkörper}

Der Bichat'sche Fettkörper wurde zur zusätzlichen Defektdeckung verwendet, wenn die Nekrose im seitlichen Oberkiefer lokalisiert war. Nach Resektion der Nekrose, wie oben beschrieben, wurde der Bichat'sche Fettkörper unterhalb des Periostes seitlich des Oberkiefers freipräpariert und über den verbliebenen Defekt gezogen. Um die Durchblutung des Fettkörpers sicherzustellten, sollte hierbei möglichst schonend vorgegangen werden. Der Fettkörper wurde mit absorbierbaren Nähten am palatinalen Periost befestigt, um ihn in Position zu halten. Die Prozedur wurde durch einen spannungsfreien und speicheldichten Wundverschluss abgeschlossen (Ristow et al., 2018).

\section{Statistik}

Die statistischen Analysen wurden mit IBM SPSS Statistics Software 25 (SPSS Inc.) und Microsoft Excel durchgeführt. Ein p-Wert von $\leq 0.05$ wurde als statistisch signifikant definiert. 


\section{ERGEBNISSE}

Zwischen September 2016 und September 2018 wurden 44 Fälle mit MRONJ im Stadium III in die Studie eingeschlossen. Klinische Parameter der Patienten sind in Tabelle 1 zusammengefasst. Das mittlere Alter unserer Patienten lag bei 68,1 Jahre (range 40-88), die durchschnittliche Behandlungszeit der antiresorptiven Therapien betrug 56,4 Monate (range 4-360). Die Mehrzahl der MRONJ-Fälle war in der Mandibula lokalisiert ( $\mathrm{n}=35)$, nur 9 Fälle in der Maxilla.

Während der Nachsorgeperiode entwickelten 12 Patienten ein Rezidiv mit freiliegendem Knochen. Wurden der Musculus mylohyoideus oder der Bichat'sche Fettkörper zur Defektdeckung verwendet, konnte ein signifikant besseres Ergebnis bezüglich der Rezidivhäufigkeit erzielt werden (OR $=0.022$; 95-CI: 0.003-0.154; $\mathrm{p}<0.001)$ (Tabelle 2; Abbildung 2).

Bei alleiniger Verwendung des Mukoperiostlappens blieben nur 2 von 11 Fälle ohne Rezidiv. Im Vergleich dazu konnte in 30 von 33 Fällen mit zusätzlicher Defektdeckung mit Musculus mylohyoideus oder Bichat'schem Fettkörper der primäre Endpunkt erreicht werden. Am Ende unserer Studie zeigten 6 von 44 Patienten exponierten Knochen.

Bezüglich der Lokalisation im Kieferknochen war das Operationsergebnis besser, wenn die Knochennekrose im hinteren Bereich lag $(\mathrm{OR}=6.905$, CI: $1.323-36.035 ; \mathrm{p}=0.025)$, ein Unterschied zwischen Ober- und Unterkieferknochen war allerdings nicht zu beobachten $(\mathrm{p}=0.531)$

Das Auftreten eines Rezidivs korrelierte nicht mit dem klinischen Durchmesser $(p=0.73)$ oder mit der radiologischen Tiefenausdehnung $(\mathrm{p}=0.35)$ der Nekrose. Auch ein Zusammenhang von operativem Erfolg und der Dauer der antiresorptiven Therapie $(p=0.315)$ oder dem Bestehen der Knochennekrose bis zur operativen Resektion $(p=0.129)$ (Tabelle 2) konnte nicht bestätigt werden. 
Während der ersten 14 Tage nach der Operation zeigten 22 von 44 Fällen Wundheilungsstörungen einschließlich der Fälle mit Rezidiv. Nach der sekundären Wundheilung wurde in 16 Fällen mit einer durchschnittlichen Heilungszeit von 99,2 Tagen (range 29-329) eine intakte Schleimhaut erreicht.

Auch in der Wundheilung konnten signifikante Unterschiede zwischen den verschiedenen chirurgischen Prozeduren beobachtet werden. Wurden der Musculus mylohyoideus oder der Bichat'sche Fettkörper zur Defektdeckung verwendet, führte das zu einem größeren Anteil an primärem und sekundärem Wundverschluss (OR $=0.057$; 95-CI: 0.006-0.503; $\mathrm{p}=0.002)$ : In dieser Gruppe zeigten nur 12 Fälle eine insuffiziente Wundheilung. Nach sekundärer Wundheilung wiesen hier 31 Fälle eine intakte und reizlose Schleimhaut auf (Abbildung 3).

Insgesamt konnte durch die chirurgische Intervention eine signifikante Schmerzreduktion erreicht werden $(\mathrm{p}<0.001)$. Präoperativ gaben die Patienten ein durchschnittliches Schmerzlevel von 3.54/10 auf einer numerischen Schmerzskala an, postoperativ lag das durchschnittliche Schmerzlevel bei 0.93/10 (Tabelle 3). Zudem klagten vor der Operation 19 von 44 Patienten über Halitosis, postoperativ gab kein Patient mehr an, unter Halitosis zu leiden $(\mathrm{p}<0.001)$. Bei einigen Patienten traten Operationsrisiken auf. 18 Patienten klagten postoperativ über eine partielle Taubheit der Lippe. Sensibilitätsstörungen der Zunge konnten nicht verzeichnet werden. In den ersten Wochen nach der Operation hat sich die prothetische Versorgung signifikant verschlechtert $\quad(p=0.001)$, auf lange Sicht lag jedoch kein signifikanter Unterschied zur präoperativen Versorgung mehr vor (Tabelle 3). 


\section{DISKUSSION}

Diese Studie wies hohe Erfolgsraten in der operativen Versorgung von Patienten mit fortgeschrittenen medikamenten-assoziierten Kiefernekrosen mittels Defektdeckung durch Verwendung des Musculus mylohyoideus und des Bichat'schen Fettkörpers auf.

Heutzutage ist die Resektion von medikamenten-assoziierten Kiefernekrosen mit einem anschließenden spannungsfreien Wundverschluss durch den Mukoperiostlappen eine etablierte chirurgische Strategie. Im Hinblick auf diese Prozedur zeigten viele Studien unbefriedigende Ergebnisse mit hohen Rezidivraten und Wundheilungsstörungen (Bodem et al., 2016; Klingelhöffer et al., 2016; Reich et al., 2015; Wutzl et al., 2008). Um bei betroffenen Patienten das bestmögliche Ergebnis zu erzielen, ist es notwendig, alternative Operationsmethoden zu testen.

Die Defektdeckung mittels Musculus mylohyoideus erreichte in neueren Studien bereits vielversprechende Ergebnisse und scheint aufgrund der zusätzlichen Schicht eines gut durchbluteten Gewebes eine verbesserte Chance auf postoperative Heilung zu bieten (Ristow et al., 2018; Mücke et al., 2016). Es existiert momentan keine ausreichende Evidenz für die Wirksamkeit dieser Therapie, vor allem bezüglich fortgeschrittener Nekrosen, da viele Studien keine oder nur wenige dieser Fälle einschließen.

Zuerst beschrieben Eckardt et al. und Lemound et al. die Defektdeckung mit einem MusculusMylohyoideus-Lappen bei Patienten mit MRONJ (Eckardt et al., 2011; Lemound et al., 2012). In der Studie von Lemound et al. lag die Erfolgsrate - hier ein vollständiger Wundverschluss des Lappens bei 90 \% (18/20). Leider wurden Patienten mit einer MRONJ im Stadium III nicht in diese Studie eingeschlossen (Lemound et al., 2012).

Ristow et al. konnten bei 57 Patienten in einem Kollektiv von 68 MRONJ-Fällen die Deckung mit einem Musculus-Mylohyoideus-Lappen durchführen. 31 Läsionen wurden als Stadium I, 26 Läsionen als Stadium II und 11 Läsionen als Stadium III klassifiziert. Am letzten Kontrolltermin, 8 Monate postoperativ, hatten 44 von 50 Patienten eine intakte Schleimhaut. 
Allerdings sind bezogen auf den chirurgischen Erfolg deutliche Unterschiede zwischen frühen (Stadium I, Stadium II) und fortgeschrittenen (Stadium III) Kiefernekrosen zu sehen. Fünf von sechs Patienten, die postoperativ keine intakte Schleimhaut vorweisen konnten, befanden sich präoperativ im Stadium III. Das führt in dieser Gruppe zu deutlich niedrigeren Erfolgsraten (Ristow et al., 2018) und zeigt die besondere Herausforderung der Behandlung von Patienten mit fortgeschrittenen medikamenten-assoziierten Kiefernekrosen.

Aus diesem Grund haben wir uns in unserer Studie besonders auf Patienten mit MRONJ im Stadium III fokussiert und konnten vielversprechende Ergebnisse durch die Verwendung des Musculus-Mylohyoideus-Lappes erreichen. Der Lappen wurde verwendet, wenn die Nekrose im lateralen Teil der Mandibula lokalisiert und der Musculus mylohyoideus dick genug für die endsprechende Präparation war. Waren diese Voraussetzungen nicht erfüllt, verwendeten wir den Mukoperiostlappen alleine. Während der Nachsorgeperiode blieben 23 von 26 Fällen, bei denen der Musculus-Mylohyoideus-Lappen angewendet wurde, rezidiv-frei. In 8 Fällen verzeichneten wir eine Wundheilungsstörung ohne freiliegenden Knochen. Bei Abschluss der Studie, nach Ablauf der sekundären Wundheilung, zeigten 24 von 26 Patienten, bei denen der Musculus-Mylohyoideus-Lappen verwendet wurde, eine intakte Schleimhaut ohne Entzündungszeichen.

War die Nekrose im lateralen Oberkiefer lokalisiert, wurde der Bichat'sche Fettkörper zur Defektdeckung präpariert. Die Vereinbarkeit von der Größe des Fettkörpers zur Ausdehnung der Nekrose war die Voraussetzung für diese Technik. Andernfalls wurde ebenfalls nur der Mukoperiostlappen verwendet.

Der Bichat'sche Fettkörper wurde im Jahr 1977 erstmals als Mittel der Defektdeckung erwähnt und hat sich bereits in Fällen von Mund-Antrum-Verbindungen bewährt (Dolanmaz et al., 2004; Egyedi, 1977; Park et al., 2019; Poeschl et al., 2009). Bezüglich medikamentenassoziierter Kiefernekrosen der Maxilla zeigen viele neuere Studien hohe Erfolgsraten bei der Verwendung des Bichat'schen Fettkörpers. Aljohani et al. konnten durch diese Prozedur in 12 von 14 Fällen von MRONJ eine intakte Schleimhaut erreichen. 13 dieser Fälle wiesen eine Mund-Antrum-Verbindung und somit, nach Klassifikation der AAOMS, eine Osteonekrose im Stadium III auf (Aljohani et al., 2019; Ruggiero et al., 2014). Unter Einschluss aller MRONJ-Stadien kam die Studie von Ristow et al. auf eine Erfolgsrate von 93,1\% (27/29) 
(Ristow et al., 2018). In unserer Studie konnten nur sieben Fälle eingeschlossen werden, bei denen der Bichat'sche Fettkörper zur Defektdeckung verwendet wurde. Trotz dieser geringen Fallzahl ist zu bedenken, dass diese Gruppe eine Erfolgsrate von 100\% aufwies und damit vergleichbare Ergebnisse wie frühere Studien mit bis zu 10 Fällen erreicht (Berrone et al., 2015; Gallego et al., 2012; Rotaru et al., 2015).

Patienten, die nur mit dem Mukoperiostlappen behandelt wurden $(n=11)$, wiesen verglichen mit Patienten, bei denen der Musculus-Mylohyoideus-Lappen oder der Bichat'sche Fettkörper zum Einsatz kam, ein signifikant schlechteres Outcome auf. Bei alleiniger Verwendung des Mukoperiostlappens traten mehr Rezidive auf $(\mathrm{p}<0.001)$, nur in 6 dieser Fälle konnte am Ende unserer Studie eine intakte Schleimhaut erreicht werden.

Die Ergebnisse von Mücke et al. werden durch unsere Daten bestätigt. Im Vergleich mit der alleinigen Verwendung des Mucoperiostlappens $(n=169)$ stellen Mücke et al. ebenfalls eine Reduktion des Rezidivrisikos durch die zusätzliche Verwendung des MusculusMylohyoideus-Lappens (n=20) heraus (Mücke et al., 2016).

Neben dem chirurgischen Erfolg ist die Verbesserung der Lebensqualität ein wichtiger Aspekt der Behandlung der MRONJ. Die meisten Fälle medikamenten-assoziierter Kiefernekrosen gehen mit einer antiresorptiven Therapie aufgrund von malignen Grunderkrankungen einher. Diese führen an sich schon zu häufigen prolongierten Krankenhausaufenthalten. Trotz der vielversprechenden Ergebnisse dieser Studie muss der lange Heilungsprozess vieler Patienten bedacht werden. Im Falle einer vorübergehenden Wundheilungsstörung (16/44) betrug die Zeit bis zum Erreichen einer intakten Mukosa zwischen 29 und 329 Tage, was eine durchschnittliche Zeit von 99,2 Tage bedeutet. Eine intensive Nachsorge scheint für ein bestmögliches Resultat für jeden Patienten und zur Vermeidung wiederholter Operationen erforderlich.

Die meisten Patienten mit fortgeschrittener Kiefernekrose leiden unter Schmerzen, Problemen beim Kauen und Halitosis. Durch unsere dargestellten chirurgischen Interventionen konnte eine durchschnittliche Schmerzreduktion von 3,54/10 auf 0,93/10 auf einer numerischen Schmerzskala erreicht werden. Zudem klagte postoperativ kein Patient mehr über Halitosis.

Bezüglich der prothetischen Versorgung wurde unsererseits befürchtet, dass durch die Verwendung des Musculus-Mylohyoideus-Lappens und die dadurch entstehende Straffung 
des Mundbodens ein herausnehmbarer Zahnersatz nicht suffizient verwendete werden könnte. Von unseren Patienten, bei denen der Musculus-Mylohyoideus-Lappen verwendet wurde, waren präoperativ 17 von 26 prothetisch versorgt. Postoperativ konnten, nach einer vorübergehenden Verschlechterung der prothetischen Versorgung während der Wundheilung, 18 von 26 Patienten erfolgreich prothetisch versorgt werden. Folglich konnte langfristig durch die Verwendung des Musculus-Mylohyoideus-Lappens zwar keine Verbesserung der prothetischen Versorgung erzielt werden, jedoch trat auch keine Verschlechterung ein. Mehr Untersuchungen bezüglich der langfristigen prothetischen Versorgung in Verbindung mit dem Musculus-Mylohyoideus-Lappen wären hier hilfreich.

Trotz vielversprechender Ergebnisse nach Anwendung des Musculus-Mylohyoideus-Lappens und der Verwendung des Bichat'schen Fettkörpers zur Defektdeckung sind in dieser Studie einige Limitierungen $\mathrm{zu}$ sehen. Die Lappen konnten nur bei entsprechenden anatomischen Gegebenheiten verwendet werden. Vor allem, wenn der Musculus mylohyoideus zu dünn für eine Präparation war, war nur die Defektdeckung mit einem Mukoperiostlappen alleine möglich. Entsprechend verlief auch die Auswahl der verschiedenen Techniken nicht randomisiert. Zudem befanden sich zwar alle Patienten im Stadium III der medikamentenassoziierte Kiefernekrose, der jeweilige Gesundheitszustand war jedoch variabel. Bei vielen Patienten lag eine maligne Grunderkrankung vor, die $\mathrm{zu}$ einer Beeinflussung der Wundheilung geführt haben könnte. 


\section{FAZIT}

Wir haben mit dieser Studie herausgefunden, dass durch die Verwendung des Musculus mylohyoideus und des Bichat'schen Fettkörpers zur zusätzlichen Defektdeckung in Fällen fortgeschrittener medikamenten-assoziierter Kiefernekrosen bessere postoperative Resultate erzielt werden konnten, als durch die alleinige Verwendung des Mukoperiostlappens. Diese zusätzliche Defektdeckung stellt daher eine gute Alternative zur gewohnten Weichteildeckung dar. Trotz ausgedehnter Heilungsprozesse, konnten die oralen Bedingungen in vielen Aspekten verbessert werden.

Die Verwendung des Musculus mylohyoideus und des Bichat'schen Fettkörpers ist eine vielversprechende operative Technik in der Behandlung von Patienten mit MRONJ. Da viele Studien nur eine kleine Anzahl an Fällen einschließen konnten, insbesondere solche mit fortgeschrittenen Nekrosen, sind an dieser Stelle weitere Untersuchungen sinnvoll. 
Tabelle 1

Tabelle 2

Tabelle 3

Abbildung 1

Abbildung 2

Abbildung 3 
Tabelle 1: Klinische Parameter der 44 Patienten mit MRONJ Stadium III

\begin{tabular}{ll} 
Patientenzahl & 44 \\
\hline Geschlecht & 22 \\
Männlich & 22 \\
Weiblich & \\
\hline Lokalisation & 9 \\
Maxilla & 35 \\
Mandibula & 8 \\
Vorderer Bereich & 36 \\
Hinterer Bereich & 37 \\
\hline Maligne Grunderkrankung & 14 \\
Prostatakarzinom & 12 \\
Mammakarzinom & 8 \\
Multiples Myelom & 2 \\
Bronchialkarzinom & 1 \\
\hline Leiomyosarkom & 7 \\
\hline Benigne Grunderkrankung & 7 \\
Osteoporose & 7 \\
\hline Therapie & 4 \\
Bisphosphonate & \\
Denosumab & 32 \\
Beide & \\
& 2 \\
\hline
\end{tabular}


Tabelle 2: Vergleich Rezidiv/kein Rezidiv

\begin{tabular}{|c|c|c|c|c|c|}
\hline $\mathrm{n}(\%)$ & $\begin{array}{l}\text { Rezidiv } \\
\mathrm{n}=12\end{array}$ & $\begin{array}{l}\text { Kein Rezidiv } \\
\mathrm{n}=32\end{array}$ & $\mathrm{p}$ bivariat & OR & KI (95\%) \\
\hline $\begin{array}{l}\text { Alter, im Mittel } \\
\text { (SD) }\end{array}$ & $66.2(9.53)$ & $68.8(11.12)$ & 0.524 & & \\
\hline Lappen & $3(25.0)$ & $30(93.8)$ & $<0.001$ & 0.022 & $0.003-0.154$ \\
\hline Muskellappen & $3(25.0)$ & $23(71.9)$ & 0.007 & 0.130 & $0.029-0.595$ \\
\hline Fettlappen & $0(0.0)$ & 7 (21.9) & 0.088 & 0.676 & $\begin{array}{l}0.540-0.845 \\
b\end{array}$ \\
\hline Nikotinkonsum & $4(33.3)$ & $5(15.6)$ & 0.188 & 2.700 & $0.583-12.511$ \\
\hline Taubheit Lippe & $7(58.3)$ & $11(34.4)$ & 0.137 & 2.673 & $\begin{array}{l}0.686-10.41 \\
2\end{array}$ \\
\hline $\begin{array}{l}\text { Maligne vs. } \\
\text { benigne } \\
\text { Grunderkrankung }\end{array}$ & $11(91.7)$ & $26(81.3)$ & 0.372 & 2.538 & $\begin{array}{l}0.273-23.63 \\
8\end{array}$ \\
\hline $\begin{array}{l}\text { Maxilla vs. } \\
\text { Mandibula }\end{array}$ & $10(83.3)$ & $25(78.1)$ & 0.531 & 1.400 & $0.247-7.930$ \\
\hline $\begin{array}{l}\text { Vorderer vs. } \\
\text { hinterer Bereich }\end{array}$ & $5(41.7)$ & $3(9.4)$ & 0.025 & 6.905 & $\begin{array}{l}1.323-36.03 \\
5\end{array}$ \\
\hline $\begin{array}{l}\text { Max. klinischer } \\
\text { Durchmesser der } \\
\text { Nekrose in } \mathrm{cm} \\
\text { (SD) }\end{array}$ & $1.35(1.22)$ & $1.21(1.39)$ & 0.725 & & $-1.030-0.751$ \\
\hline $\begin{array}{l}\text { Max. } \\
\text { radiologischer } \\
\text { Durchmesser der } \\
\text { Nekrose in cm } \\
\text { (SD) }\end{array}$ & $2.47(0.525)$ & $2.66(0.761)$ & 0.348 & & $-0.221-0.608$ \\
\hline $\begin{array}{l}\text { Zeitdauer der } \\
\text { Nekrose in } \\
\text { Monaten (SD) }\end{array}$ & $21.0(18.2)$ & $11(14.4)$ & 0.129 & & $\begin{array}{l}-21.711-3.02 \\
4\end{array}$ \\
\hline $\begin{array}{l}\text { Zeitdauer der } \\
\text { antiresorptiven } \\
\text { Therapie in } \\
\text { Monaten (SD) }\end{array}$ & $77.5(92.5)$ & $48.6(38.6)$ & 0.315 & & $\begin{array}{l}-88.801-30.9 \\
89\end{array}$ \\
\hline
\end{tabular}


Tabelle 3: Parameter zur Lebensqualität

\begin{tabular}{llllll}
$\mathrm{n}(\%)$ & präoperativ & postoperativ & $\mathrm{p}$ & $\mathrm{OR}$ & $\mathrm{KI}(95 \%)$ \\
\hline Schmerz (Skala, SD) & $3.54(3.33)$ & $0.93(2.19)$ & $<0.001$ & & \\
\hline $\begin{array}{l}\text { Definitive prothetische } \\
\text { Versorgung }\end{array}$ & $26(59.1)$ & $11(25.0)$ & 0.001 & 1.733 & $1.247-2.409$ \\
früh postoperativ & & & & & \\
\end{tabular}

\begin{tabular}{llllll}
\hline Definitive prothetische & $26(59.1)$ & $31(70.5)$ & 0.213 & 0.794 & $0.520-1.214$
\end{tabular}

Versorgung

spät postoperativ

Definitive prothetische $\quad 17(65.4) \quad 7(26.9) \quad 0.03 \quad 1.700 \quad 1.142-2.530$

Versorgung

früh postoperativ

Muskellappen

\begin{tabular}{llllll}
\hline Definitive prothetische & $17(65.4)$ & $18(69.2)$ & 0.255 & 0.520 & $0.383-1.379$
\end{tabular}

Versorgung

spät postoperativ

Muskellappen

\begin{tabular}{llllll}
\hline Halitosis & $19(43.2)$ & $0(0)$ & $<0.001$ & a & a \\
\hline Taubheit Lippe & $0(0)$ & $18(40.9)$ & $<0.001$ & a & a \\
\hline Taubheit Zunge & $0(0)$ & $0(0)$ & a & &
\end{tabular}

${ }^{a}$ statistische Analyse nicht möglich 
Abbildung 1: Musculus-Mylohyoideus-Lappen

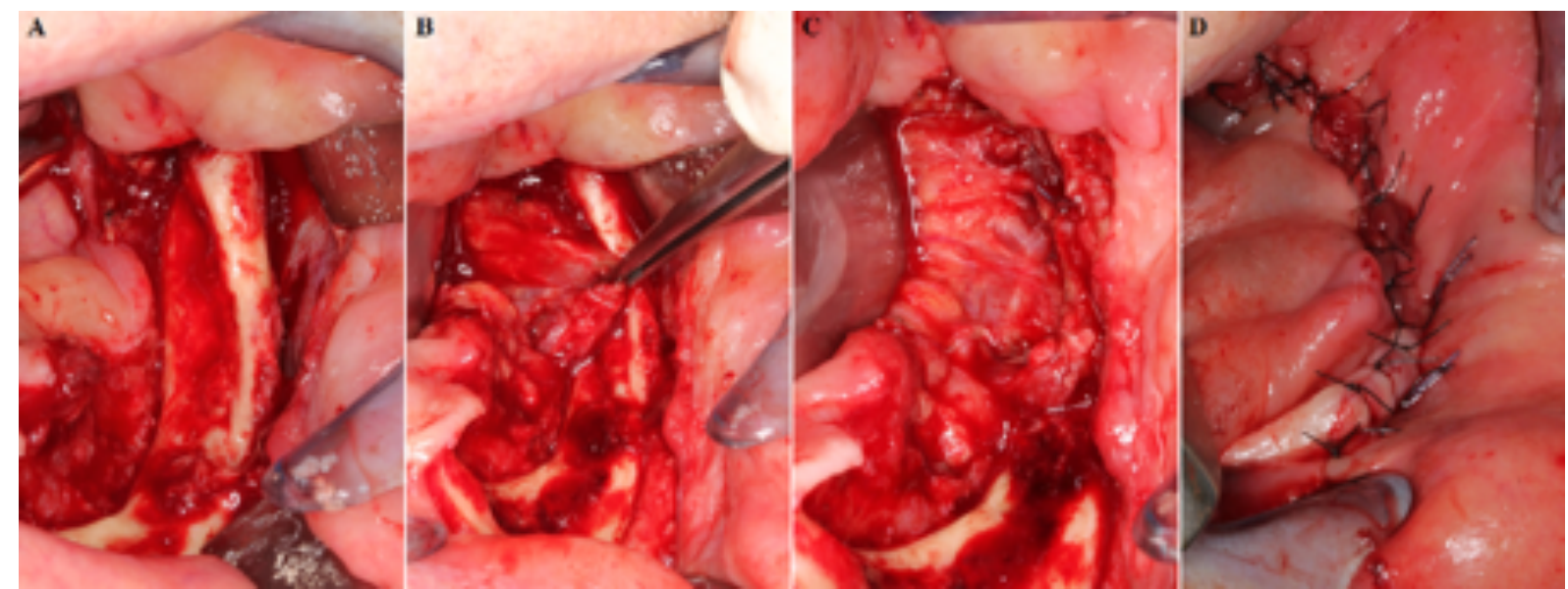

Der Musculus mylohyoideus kann bei Kiefernekrosen als Muskellappen zum Weichteilverschluss verwendet werden. Nach Entfernen des nekrotischen Knochengewebes im Unterkiefer (A) muss der Muskel präpariert, über den Unterkieferknochen gezogen (B) und am vestibulären Periost befestigt werden (C). Wichtig ist ein spannungsfreier Wundverschluss am Ende der Prozedur (D). 
Abbildung 2: MRONJ-Rezidiv mit und ohne Defektdeckung durch Musculus mylohyoideus oder Bichat' ${ }^{\text {chen Fettkörper }}$

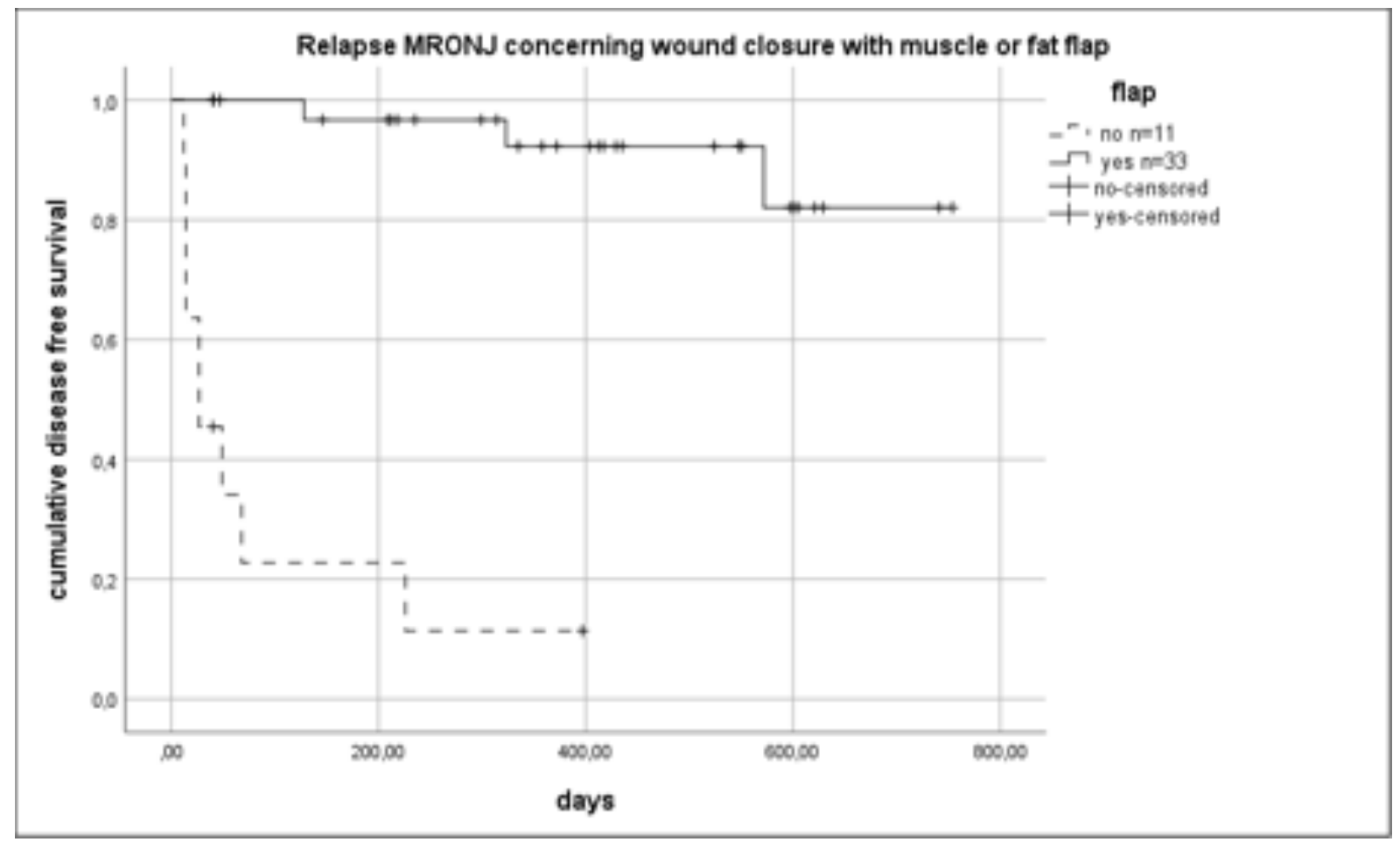


Abbildung 3: Wundheilung mit und ohne Defektdeckung durch Musculus mylohyoideus oder Bichat'schen Fettkörper

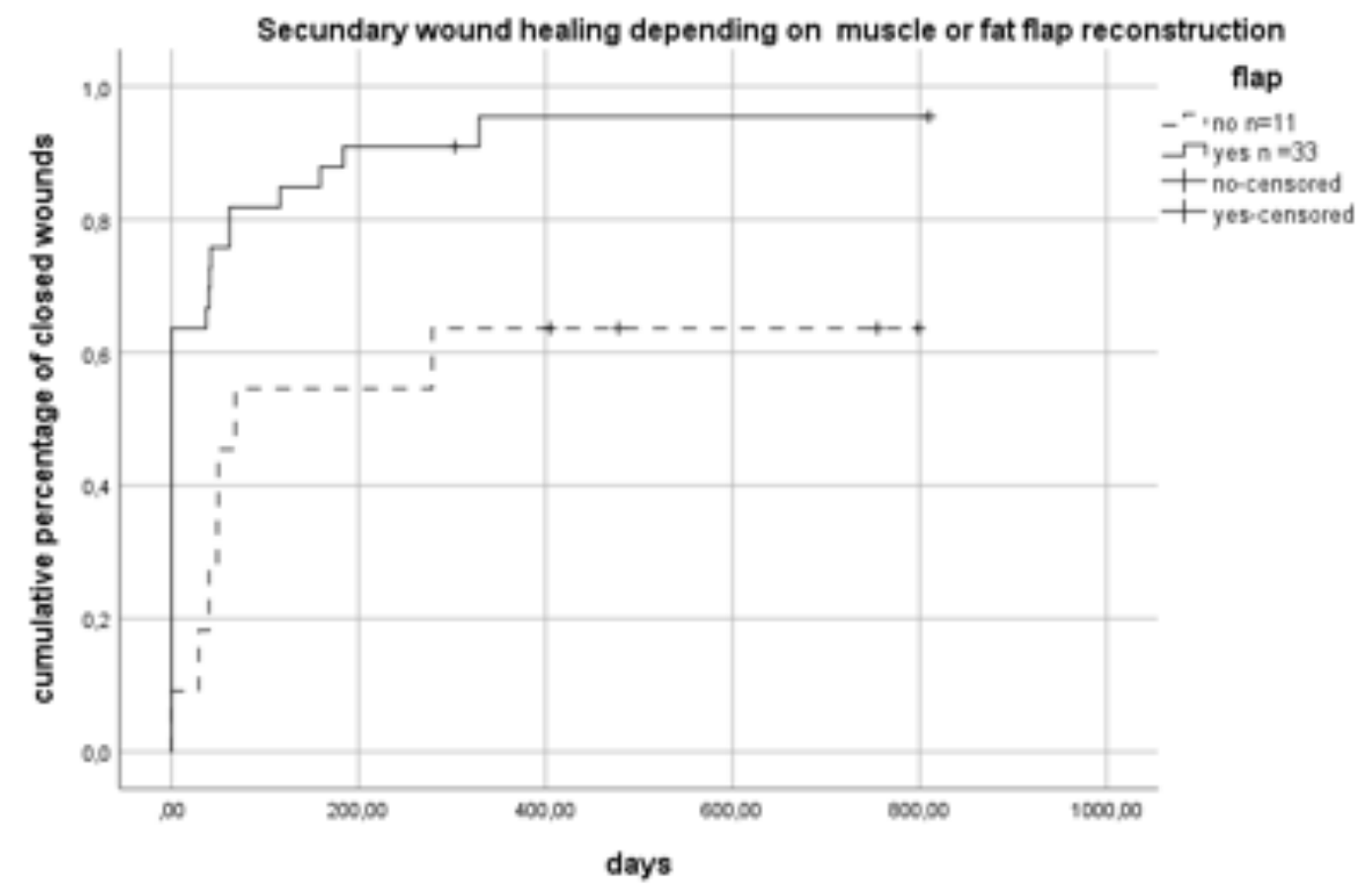




\section{REFERENZEN}

Aljohani S, Troeltzsch M, Hafner S, Kaeppler G, Mast G, Otto S: Surgical treatment of medication-related osteonecrosis of the upper jaw: Case series. Oral Dis 2019; 25(2):497-507.

Amgen Canada Inc.: Prolia Product Monograph, 2019.

Berrone M, Florindi FU, Carbone V, Aldiano C, Pentenero M: Stage 3 Medication-related osteonecrosis of the posterior maxilla: surgical treatment using a pedicled buccal fat pad flap: case reports. J Oral Maxillofac Surg 2015; 73(11):2082-6.

Bodem JP, Schaal C, Kargus S, Saure D, Mertens C, Engel M, et al.: Surgical management of bisphosphonate-related osteonecrosis of the jaw stages II and III. Oral Surg Oral Med Oral Pathol Oral Radiol 2016; 121(4):367-72.

Carlson ER: Management of antiresorptive osteonecrosis of the jaws with primary surgical resection. J Oral Maxillofac Surg 2014; 72(4):655-7.

Carlson ER, Basile JD: The role of surgical resection in the management of bisphosphonaterelated osteonecrosis of the jaws. J Oral Maxillofac Surg 2009; 67(5 Suppl):85-95.

Coleman R, Woodward E, Brown J, Cameron D, Bell R, Dodwell D, et al.: Safety of zoledronic acid and incidence of osteonecrosis of the jaw (ONJ) during adjuvant therapy in a randomised phase III trial (AZURE: BIG 01-04) for women with stage II/III breast cancer. Breast Cancer Res Treat 2011; 127(2):429-38.

Dolanmaz D, Tuz H, Bayraktar S, Metin M, Erdem E, Baykul T: Use of pedicled buccal fat pad in the closure of oroantral communication: analysis of 75 cases. Quintessence Int 2004; $35(3): 24-6$. 
Eckardt A, Lemound J, Lindhorst D, Rana M, Gellrich N-C: Surgical management of bisphosphonate-related osteonecrosis of the jaw in oncologic patients: a challenging problem. Anticancer Res 2001; 31(6):2313-8.

Egyedi P: Utilization of the buccal fat pad for closure of oro-antral and/or oro-nasal communications. J Maxillofac Surg 1977; 5(4):241-4.

Gallego L, Junquera L, Pelaz A, Hernando J, Megías J: The use of pedicled buccal fat pad combined with sequestrectomy in bisphosphonate-related osteonecrosis of the maxilla. Med Oral Patol Oral Cir Bucal 2012; 17(2):e236-41.

Graziani F, Vescovi P, Campisi G, Favia G, Gabriele M, Gaeta GM, et al.: Resective surgical approach shows a high performance in the management of advanced cases of bisphosphonaterelated osteonecrosis of the jaws: a retrospective survey of 347 cases. J Oral Maxillofac Surg 2012; 70(11):2501-7.

Hallmer F, Andersson G, Gotrick B, Warfvinge G, Anderud J, Bjornland T: Prevalence, initiating factor, and treatment outcome of medication-related osteonecrosis of the jaw-a 4year prospective study. Oral Surg Oral Med Oral Pathol Oral Radiol 2018; 126(6):477-85.

Hoff AO, Toth BB, Altundag K, Johnson MM, Warneke CL, Hu M, et al.: Frequency and risk factors associated with osteonecrosis of the jaw in cancer patients treated with intravenous bisphosphonates. J Bone Miner Res 2008; 23(6):826:36.

Klingelhöffer C, Zeman F, Meier J, Reichert TE, Ettl T: Evaluation of surgical outcome and influencing risk factors in patients with medication-related osteonecrosis of the jaws. J Craniomaxillofac Surg 2016; 44(10)1694-9.

Lee S-H, Chang S-S, Lee M, Chan R-C, Lee C-C: Risk of osteonecrosis in patients taking bisphosphonates for prevention of osteoporosis: a systematic review and meta-analysis. Osteoporos Int 2014; 25(3):1131-9.

Lemound J, Eckardt A, Kokemüller H, See C von, Voss PJ, Tavassol F, et al.: Bisphosphonate-associated osteonecrosis of the mandible: reliable soft tissue reconstruction using a local myofascial flap. Clin Oral Investig 2012; 16(4):1143-52. 
Lo JC, O'Ryan FS, Gordon NP, Yang J, Hui RL, Martin D, et al.: Prevalence of osteonecrosis of the jaw in patients with oral bisphosphonate exposure. J Oral Maxillofac Surg 2010; 68(2): 243-53.

Marx RE: Pamidronate (Aredia) and zoledronate (Zometa) induced avascular necrosis of the jaws. Journal of Oral and Maxillofacial Surgery 2003; 61(9):1115-7.

Marx RE, Sawatari Y, Fortin M, Broumand V: Bisphosphonate-induced exposed bone (osteonecrosis/osteopetrosis) of the jaws: risk factors, recognition, prevention, and treatment. J Oral Maxillofac Surg 2005; 63(11):1567-75.

Mauri D, Valachis A, Polyzos IP, Polyzos NP, Kamposioras K, Pesce LL: Osteonecrosis of the jaw and use of bisphosphonates in adjuvant breast cancer treatment: a meta-analysis. Breast Cancer Res Treat 2009; 116(3):433-9.

Mücke T, Koerdt S, Jung M, Mitchell DA, Wolff K-D, Kesting MR, et al.: The role of mylohyoid flap in the treatment of bisphosphonate-related osteonecrosis of the jaws. J Craniomaxillofac Surg 2016; 44(4):369-73.

Otto S, Schreyer C, Hafner S, Mast G, Ehrenfeld M, Stürzenbaum S, et al.: Bisphosphonaterelated osteonecrosis of the jaws - characteristics, risk factors, clinical features, localization and impact on oncological treatment. J Craniomaxillofac Surg 2012; 40(4):303-9.

Park J, Chun B-d, Kim U-K, Choi N-R, Choi H-S, Hwang D-S: Versatility of the pedicled buccal fat pad flap for the management of oroantral fistula: a retrospective study of 25 cases. Maxillofac Plast Reconstr Surg 2019; 41(1):50.

Poeschl PW, Baumann A, Russmueller G, Poeschl E, Klug C, Ewers R: Closure of oroantral communications with bichat's buccal fat pad. Journal of Oral and Maxillofacial Surgery 2009; 67(7):1460-6.

Qi W-X, Tang L-N, He A-N, Yao Y, Shen Z: Risk of osteonecrosis of the jaw in cancer patients receiving denosumab: a meta-analysis of seven randomized controlled trials. Int $\mathrm{J}$ Clin Oncol 2014; 19(2):403-10. 
Reich W, Bilkenroth U, Schubert J, Wickenhauser C, Eckert AW: Surgical treatment of bisphosphonate-associated osteonecrosis: Prognostic score and long-term results. J Craniomaxillofac Surg 2015; 43(9):1809-22.

Ristow O, Otto S, Troeltzsch M, Hohlweg-Majert B, Pautke C: Treatment perspectives for medication-related osteonecrosis of the jaw (MRONJ). J Craniomaxillofac Surg 2015; 43(2): 290-3.

Ristow O, Rückschloß T, Bodem J, Berger M, Bodem E, Kargus S, et al.: Double-layer closure techniques after bone surgery of medication-related osteonecrosis of the jaw - A single center cohort study. J Craniomaxillofac Surg 2018; 46(5):815-24.

Rotaru H, Kim M-K, Kim S-G, Park Y-W: Pedicled buccal fat pad flap as a reliable surgical strategy for the treatment of medication-related osteonecrosis of the jaw. J Oral Maxillofac Surg 2015; 73(3):437-42.

Rugani P, Walter C, Kirnbauer B, Acham S, Begus-Nahrman Y, Jakse N: Prevalence of medication-related osteonecrosis of the jaw in patients with breast cancer, prostate cancer, and multiple myeloma. Dent J (Basel) 2016; 4(4).

Ruggiero SL, Dodson TB, Assael LA, Landesberg R, Marx RE, Mehrotra B: American Association of Oral and Maxillofacial Surgeons position paper on bisphosphonate-related osteonecrosis of the jaws--2009 update. J Oral Maxillofac Surg 2009; 67(5 Suppl):2-12.

Ruggiero SL, Dodson TB, Fantasia J, Goodday R, Aghaloo T, Mehrotra B, et al.: American Association of Oral and Maxillofacial Surgeons position paper on medication-related osteonecrosis of the jaw--2014 update. J Oral Maxillofac Surg 2014; 72(10):1938-56.

Russell RGG, Watts NB, Ebetino FH, Rogers MJ: Mechanisms of action of bisphosphonates: similarities and differences and their potential influence on clinical efficacy. Osteoporos Int 2008; 19(6):733-59.

Saussez S, Javadian R, Hupin C, Magremanne M, Chantrain G, Loeb I, et al.: Bisphosphonate-related osteonecrosis of the jaw and its associated risk factors: a Belgian case series. Laryngoscope 2009; 119(2):323-9. 
Vahtsevanos K, Kyrgidis A, Verrou E, Katodritou E, Triaridis S, Andreadis CG, et al.: Longitudinal cohort study of risk factors in cancer patients of bisphosphonate-related osteonecrosis of the jaw. J Clin Oncol 2009; 27(32):5356-62.

Wilde F, Heufelder M, Winter K, Hendricks J, Frerich B, Schramm A, et al.: The role of surgical therapy in the management of intravenous bisphosphonates-related osteonecrosis of the jaw. Oral Surg Oral Med Oral Pathol Oral Radiol Endod 2011; 111(2):153-63.

Wutzl A, Biedermann E, Wanschitz F, Seemann R, Klug C, Baumann A, et al.: Treatment results of bisphosphonate-related osteonecrosis of the jaws. Head Neck 2008; 30(9):1224-30. 


\section{VERÖFFENTLICHTES MANUSKRIPT}

Am Ende der Arbeit ist das veröffentlichte Manuskript (Ahrenbog et al., 2020; Journal Impact-Fator: 1,766), welches die Grundlage dieser Dissertationsschrift bildet, beigefügt. 


\section{DANKSAGUNG}

Ich möchte mich hiermit herzlichst bei Herrn PD Dr. Dr. Christoph Klingelhöffer für die außerordentliche Betreuung und die phänomenale Zusammenarbeit während des gesamten Projekts bedanken. Von der Bereitstellung des Themas über die Datenerhebung bis zur Veröffentlichung der Arbeit stand er mir als erster Ansprechpartner stets mit konstruktiven Ratschlägen und Hilfestellungen zur Seite.

Außerdem möchte ich der Klinik und Poliklinik für Mund-, Kiefer- und Gesichtschirurgie des Universitätsklinikums Regensburg und ihrem Klinikdirektor Herrn Prof. Dr. Dr. Torsten E. Reichert sowie den Mitarbeitern Herrn Prof. Dr. Dr. Tobias Ettl, Herrn Dr. Dr. Johannes Meier und Herrn Dr. Dr. Maximilian Gottsauner für die Unterstützung und die wichtigen Beiträge zur Publikation der Studie danken.

Für die Hilfe bei der Erstellung der Statistik möchte ich mich höflichst bei Herrn Florian Zeman bedanken.

$\mathrm{Zu}$ guter Letzt gilt mein Dank auch meiner Familie und meinen Freunden, die mir auch über mein Studium hinaus stets große Unterstützung und Halt gegeben und mir ein fokussiertes Studium mit Freude und Abwechslung ermöglicht haben. 


\title{
Surgical treatment of advanced medication-related osteonecrosis of the jaws: Comparison of soft tissue closure techniques and evaluation of side effects
}

\author{
Greta Ahrenbog, Maximilian Gottsauner, Johannes K. Meier, Tobias Ettl, \\ Torsten E. Reichert, Christoph Klingelhöffer* \\ Department of Cranio-Maxillofacial Surgery, Hospital of the University of Regensburg, Franz-Josef-Strauß-Allee 11, 93053 Regensburg, Germany
}

\section{A R T I C L E I N F O}

\section{Article history:}

Paper received 18 May 2020

Accepted 25 July 2020

Available online 1 August 2020

\section{Keywords:}

MRONJ

Bisphosphonates

Mylohyoid Muscle Flap

Denosumab

Buccal Fat Flap

Surgery

\begin{abstract}
A B S T R A C T
The aim of the study was to evaluate the surgical outcome of patients with advanced medication-related osteonecrosis of the jaw (MRONJ) treated with different flaps for soft tissue closure. Only MRONJ stage III patients with a minimum follow-up of 6 months were included in our prospective study. The soft tissue closure techniques were: Mylohyoid Muscle Flap, Buccal Fat Flap and mucoperiosteal flap alone. Potential risk factors and surgical side effects were analyzed. Relapses occurred in 12 of 44 included cases and 38 reached mucosal integrity within the follow-up. Cases treated with the muscle or fat flap showed better results regarding the recurrence rate $(p<0.001)$ and soft tissue healing $(p=0.002)$ : only 3 of 33 developed a relapse, and 31 of 33 reached mucosal integrity. The outcome was worse if MRONJ occurred at the front areas of the jaw $(\mathrm{p}=0.025)$. Postoperative, the pain level was reduced significantly $(\mathrm{p}<$ 0.001 ). Partial hypoesthesia of the lip arose in 18 cases. An impairment of the long-term prosthetic rehabilitation has not been seen.Patients with MRONJ stage III undergoing surgery benefit from extensive soft tissue closure.
\end{abstract}

() 2020 European Association for Cranio-Maxillo-Facial Surgery. Published by Elsevier Ltd. All rights reserved.

\section{Introduction}

Medication-related osteonecrosis of the jaw (MRONJ) is an adverse reaction of antiresorptive drugs like Bisphosphonates or Denosumab (Amgen Canada Inc., 2019; Lee et al., 2014; Marx, 2003). These drugs inhibit osteoclasts and thereby the bone resorption (Amgen Canada Inc., 2019; Russell et al., 2008). Most frequent reasons for an antiresorptive therapy are bone-consuming malignant diseases and metastasis of the bone, but also benign disease like osteoporosis (Otto et al., 2012; Saussez et al., 2009). How antiresorptive therapy leads to osteonecrosis of the jaw is not fully understood. The prevalence of MRONJ ranges between $0.0043 \%$ and $5.16 \%$ and seems to depend on the type and dose of the drug and the underlying disease (Coleman et al., 2011; Hallmer et al., 2018; Lo et al., 2010; Mauri et al., 2009; Rugani et al., 2016; Qi et al., 2014). Local factors like poor oral health or tooth extractions seem to increase the chance of developing MRONJ (Hoff et al.,

\footnotetext{
* Corresponding author.

E-mail address: christoph.klingelhoeffer@ukr.de (C. Klingelhöffer).
}

2008; Vahtsevanos et al., 2009). Therefore, prevention is an important factor regarding the management of MRONJ (Hoff et al., 2008; Marx et al., 2005).

Since the side effect was first mentioned in 2003, experts have been discussing the best stage-dependent treatment (Bodem et al., 2016; Carlson, 2014; Marx, 2003; Ristow et al., 2015). For initial stages of MRONJ the American Association of Oral and Maxillofacial Surgery (AAOMS) recommends a conservative treatment consisting of a scheduled follow-up, antibacterial mouth rinsing, pain killers, an oral antibiotic therapy and/or a local debridement in cases of infection (Ruggiero et al., 2014). Surgery, as a standardized therapy, is recommended for patients with advanced necrosis, who suffer from extraoral fistulas, oroantral or oronasal communication, extending osteolysis or even pathologic fractures (Ruggiero et al., 2014).

The well-established surgical procedure in cases of MRONJ basically consists of (1) a marginal, segmental or continuity resection of necrotic and infected bone until sufficient bone bleeding, (2) smoothening of sharp bone margin and (3) a mucoperiosteal flap for a tension-free wound closure. This procedure shows a wide range regarding a positive outcome meaning as complete mucosal 
healing: 27.6\% (Klingelhöffer et al., 2016); 43.8\% (Reich et al., 2015); 51.1\% (Bodem et al., 2016); 68\% (Graziani et al., 2012); 88\% (Wilde et al., 2011); 91.6\% (Carlson and Basile, 2009). Some studies have shown, that a modification in tissue-closure can lead to high surgical outcomes (Mücke et al., 2016; Ristow et al., 2018; Wutzl et al., 2008).

The aim of this study was to test the Mylohyoid Muscle Flap and the Buccal Fat Flap as additional tissue closure in advanced cases of MRONJ compared to mucoperiosteal flap closure alone. Additionally, influencing factors, postoperative risks and the patients' pain levels were evaluated.

\section{Material and methods}

\subsection{Patients and data collection}

This study was approved by the local ethical committee (Nr. 16101-0257). Between September 2016 and March 2019, the prospective monocentric cohort observation study was conducted by the Department of Oral and Maxillofacial Surgery of the University Hospital of Regensburg. Using the classification of the American Association of Oral and Maxillofacial Surgeons (AAOMS) of 2009 and 2014 only MRONJ stage III cases were included in our study (Ruggiero et al., 2009, 2014). Inclusion criteria consist of the existence of a MRONJ stage III as defined by the AAOMS, a surgical treatment and a follow-up period of at least 6 months. The following criteria led to exclusion: history of radiation therapy of the head or neck region, metastatic disease within the jawbone, a therapy that differs from the ones described below.

Preoperatively we collected several clinical parameters. By clinical inspection localization of MRONJ was categorized in front (between canines) and back (canine region till retromolar region) areas. Upper and lower jaw were differentiated as well. Furthermore, clinical maximal diameter of exposed bone was measured during inspection. Depth of MRONJ was evaluated at the preoperative panoramic radiograph by measuring from bone surface to the deepest point of osteolysis. Patients estimated their pain level on a scale from 0 (no pain) to 10 (maximum imaginable pain). The existence of halitosis was asked. Prosthetic rehabilitation was evaluated and defined as definitive if patients were using sufficient dentures or all supporting zone of teeth were well preserved. Sensation of the lip and tongue were clinically tested and numbness was considered if sensation was reduced in comparison to an unaffected site.

The primary endpoint was defined as a mucosal integrity (no exposed bone) without signs of inflammation or relapse of MRONJ. Swelling, fistulas, pus, and reddish mucosa were considered as signs of inflammation. Relapse was defined as recurrence of exposed bone within the operation area.

\subsection{Surgical treatment}

All patients were hospitalized for 10 days. In general anesthesia, necrotic areas were resected with a small margin of visible vital bone by a bone saw and piezo surgery. After removal of the resected bone, sharp bone edges and the surface were smoothed till visible bleeding was reached. Afterwards, wound closure was performed by using different techniques as described below. Patients received a perioperative intravenous antibiotic therapy with Amoxicillin and Clavulanic Acid during the stay. In case of a documented allergic reaction against penicillin the patients were treated with Clindamycin. Furthermore, an antimicrobial mouth rinse with chlorhexidine (CHX) (0.12\%) was used three times a day. All patients got a postoperative nasogastric feeding tube. Suture removal was performed 14 days after surgery.

A standardized follow-up period was determined in our outpatients' clinic: 14 days post-operative; 6 weeks post-operative; every 3 months within the first year after surgery, then every 6 months. The minimum follow-up was 6 months and the median followup was 13.2 months. Additionally, panoramic radiographic images were conducted 6 months after surgery.

\subsection{Mylohyoid Muscle Flap}

The Mylohyoid Muscle Flap was used for tissue closure after the resection of osteonecrosis of the lower jaw. The mylohyoid muscle has to be freed from the mylohyoid line, and needs to be separated from mucosa and the periosteum and pulled over the remaining mandible while preserving the integrity of the lingual nerve and the submandibular duct. A tension-free fixation of the muscle at the vestibular periosteum is important (Lemound et al., 2012; Ristow et al., 2018). An additional mucoperiosteal flap completes the sufficient wound closure (Fig. 1). If the mylohyoid muscle was too thin for dissection or the defect was located unfavorably (anterior jaw area) the wound closure was assured with only a mucoperiosteal flap.

\subsection{Buccal Fat Flap}

If the resection of osteonecrosis was performed in the lateral upper jaw, the Buccal Fat Flap was used for tissue closure. A subperiosteal dissection along the buccal-sulcus leads to the necrotic area and clears the way for the Buccal Fat Flap. After the removal of necrotic areas the Buccal Fat Flap has to be separated from the lateral pterygoid muscle and pulled over the remaining defect. To
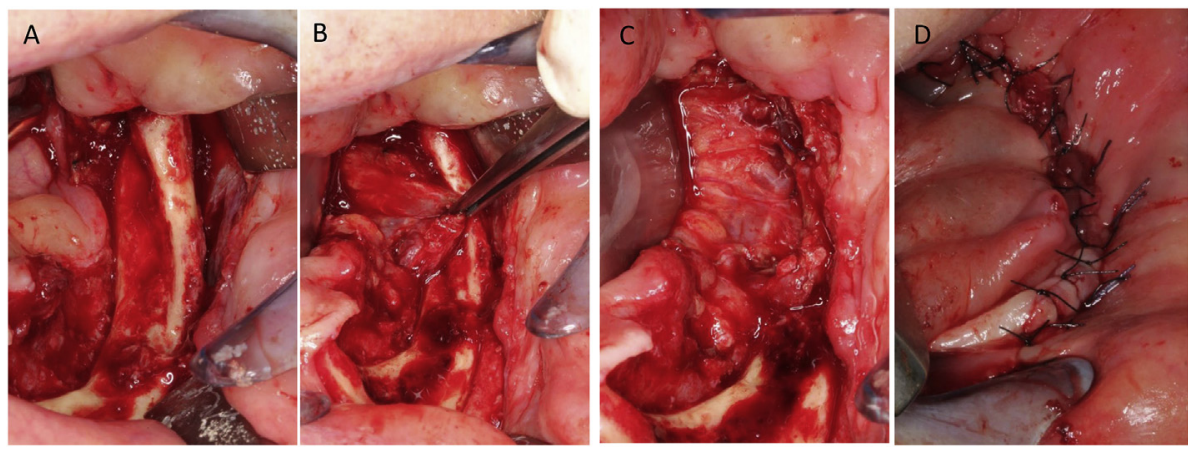

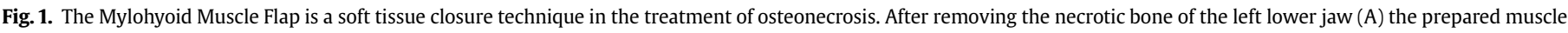
must be pulled over the jawbone (B) and fixed at the vestibular periosteum (C). A tension-free wound closure at the end of the procedure is important (D). 
preserve the internal maxillary artery, one should not cut into the fat pad itself. The flap should be fixed at the palatine periosteum with absorbable suture material for holding it in position. A tension-free and saliva-tight wound closure completes the procedure (Ristow et al., 2018).

\subsection{Statistics}

Statistical analysis was made with IBM SPSS Statistics Software 25 (SPSS Inc.) and Microsoft Excel. A p-value $\leq 0.05$ was defined as statistically significant.

\section{Results}

Between September 2016 and September 2018, we included 44 cases of MRONJ stage III in our study. Patients' characteristics are provided in Table 1 . The mean age of our patients was 68.1 years (range 40-88), the mean duration of treatment with antiresorptive therapy was 56.4 months (range 4-360). Most of the cases were located in the mandible $(\mathrm{n}=35)$, only 9 were located in the maxilla.

During the follow-up period 12 patients developed a relapse with exposed bone. The use of the Mylohyoid Muscle Flap or the Buccal Fat Flap showed a significantly better outcome regarding the recurrence of MRONJ (OR $=0.022 ; 95-\mathrm{CI}$ : 0.003-0.154; $\mathrm{p}<0.001$ ) (Fig. 2) In 30 of 33 flap cases the first endpoint was reached. In comparison, just 2 of the 11 cases where only a mucoperiosteal flap was performed have been free of recurrence. At the end of our study 6 of 44 patients still showed exposed bone.

The surgical outcome was better when MRONJ was located in the back of the jaw than in the front $(\mathrm{OR}=6.905, \mathrm{CI}$ : $1.323-36.035$; $\mathrm{p}=0.025)$, but there was no difference between the upper and the lower jaw $(\mathrm{p}=0.531)$.

There was no significant relation between the appearance of a relapse and either the maximum clinical $(p=0.73)$ or radiologic depth extension $(p=0.35)$. Also, the operative success did not depend on the duration of the necrosis until surgery $(p=0.129)$ or the duration of antiresorptive therapy $(\mathrm{p}=0.315)$ (Table 2$)$.

Within 14 days after surgery 22 of the 44 cases showed insufficiency of soft tissue healing, including the cases of relapse. After secondary wound healing 16 reached mucosal closure with a mean duration of 99.2 days (range 29-329).

Table 1

Clinical parameters of 44 patients with MRONJ stage III.

\begin{tabular}{ll}
\hline & $\mathrm{n}$ \\
\hline Number of patients & 44 \\
Sex & \\
Male & 22 \\
Female & 22 \\
Localization & \\
Maxilla & 9 \\
Mandible & 35 \\
Front & 8 \\
Back & 36 \\
Primary malignant disease & 37 \\
Prostate carcinoma & 14 \\
Mamma carcinoma & 12 \\
Multiple myeloma & 8 \\
Bronchial carcinoma & 2 \\
Leomyosarcoma & 1 \\
Primary benign disease & 7 \\
Osteoporosis & 7 \\
Therapy & \\
Bisphosphonate & 32 \\
Denosumab & 4 \\
Both & 8 \\
\hline
\end{tabular}

There were significant differences comparing the success of the different surgical procedures. The use of Mylohyoid Muscle Flap or Fat Flap (33 cases) led to a higher amount of sufficient early and late mucosal closure $(\mathrm{OR}=0.057 ; 95-\mathrm{CI}$ : 0.006-0.503; $\mathrm{p}=0.002)$ : in this group only 12 developed insufficiency of soft tissue healing and after secondary wound healing 31 reached full mucosal closure (Fig. 3).

A significant pain reduction was observed ( $\mathrm{p}<0.001$ ). Preoperative patients quoted an average pain level of 3.54/10 on a numerical pain rating scale, postoperative the average pain level has been reduced to $0.93 / 10$ (Table 3 ). Before surgery 19 of the 44 patients were complaining of halitosis, this was reduced to 0 postoperatively. Apparently, some patients suffered from secondary effects of the surgery. Eighteen complained of a partial hypoesthesia of the lip after surgery. No impairment of the tongue nerve was detected. Although the prosthetic maintenance has been significantly degraded within the first weeks after surgery $(p=0.001)$, the prosthetic rehabilitation was successful in the long run (Table 3).

\section{Discussion}

This study provided high success rates in the surgical outcome of patients with advanced MRONJ by using the Mylohyoid Muscle Flap and the Buccal Fat Flap for wound closure.

Nowadays, the resection of necrotic bone and a subsequent mucoperiosteal flap for a tension-free wound closure is wellestablished in the surgical treatment of MRONJ. With respect to this procedure, many studies show non-satisfying results with a high number of relapses and wound insufficiencies (Bodem et al., 2016; Klingelhöffer et al., 2016; Reich et al., 2015; Wutzl et al., 2008). For the best outcome of affected patients, it is necessary to test alternative surgical procedures.

The Mylohyoid Muscle Flap achieved promising results in some of the latest studies and seems to provide a better chance of postoperative healing due to the extra layer of well-vascularized soft tissue. So far, it is difficult to render evidence, especially regarding patients with advanced necrosis, since many studies include none or just a small amount of these cases.

After the Mylohyoid Muscle Flap was mentioned first by Eckardt et al. and Lemound et al. in relation to MRONJ, to the best of our knowledge, they presented the technique in detail in their following study (Eckardt et al., 2011; Lemound et al., 2012). A success rate of $90 \%(18 / 20)$ meaning full mucosal closure was shown using the flap. Unfortunately, stage III patients are missing here (Lemound et al., 2012). In the study by Ristow et al. 57 patients with 68 lesions of MRONJ were treated with the Mylohyoid Muscle Flap. Thirty-one lesions were classified as stage I, 26 lesions as stage II and 11 lesions as stage III. At the last time point, eight months after surgery, a mucosal integrity in 44 of 50 patients has been registered. But one must keep in mind that clearly differences can be seen between the surgical outcome of advanced (stage III) and earlier (stage I and stage II) necrosis. Five of the six patients without mucosal integrity are related to stage III, which leads to a lower success rate in this group (Ristow et al., 2018). The outcome of this study illustrates the outstanding challenge with reference to the treatment of advanced cases.

Therefore, we focused on patients with MRONJ stage III in this study and are able to fortify the promising role of the Mylohyoid Muscle Flap for these patients. The flap has been used, if the necrosis was located in the lateral part of the lower jaw and if the mylohyoid muscle was thick enough for the preparation. If the requirements were not met, the mucoperiosteal flap has been performed alone. During the follow-up period, 23 of 26 cases treated with the Mylohyoid Muscle Flap were free of relapse. Besides, 8 


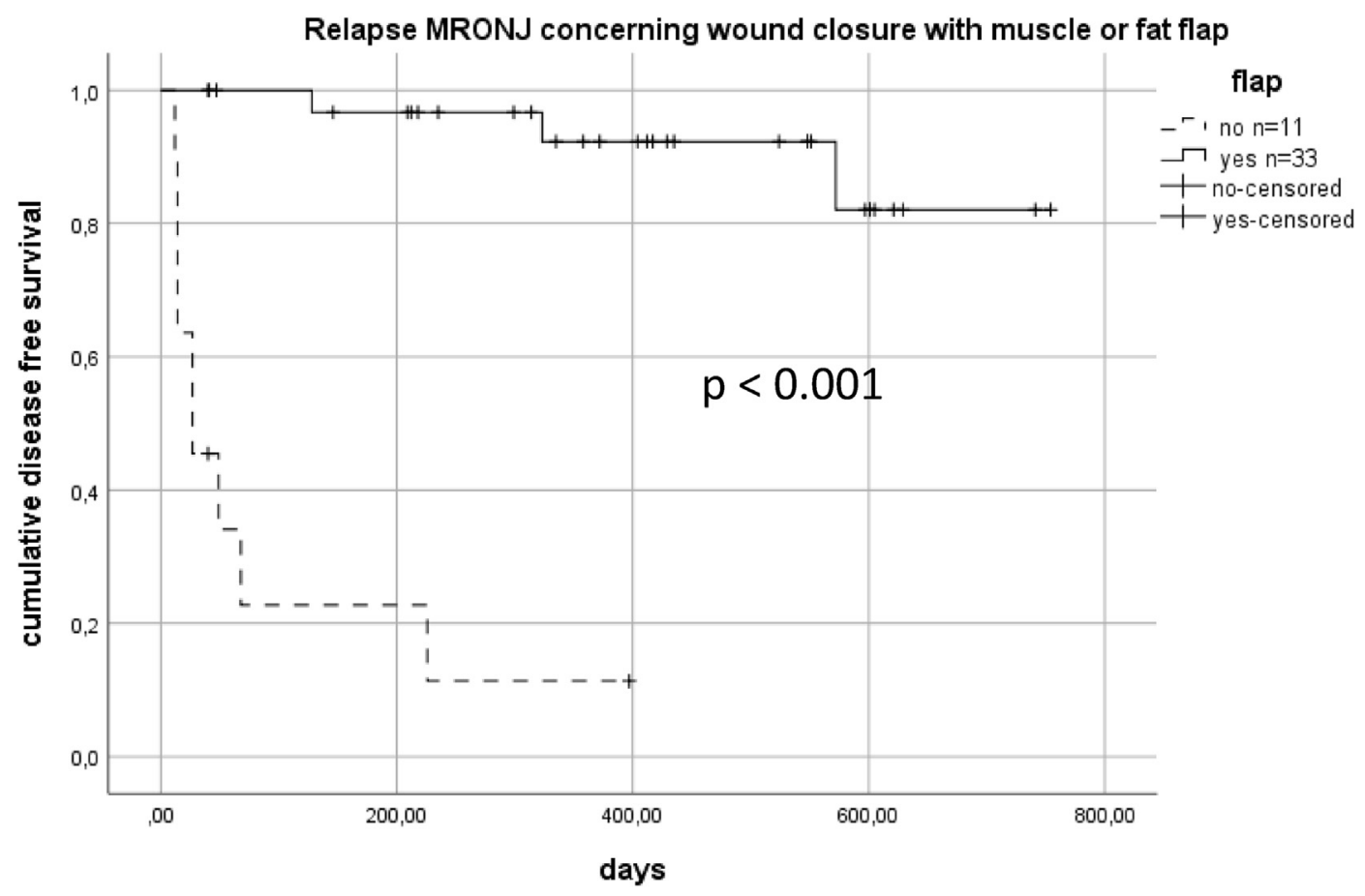

Fig. 2. Relapse MRONJ concerning wound closure with muscle or fat flap.

Table 2

Influencing factors of MRONJ relapse.

\begin{tabular}{|c|c|c|c|c|c|}
\hline$n$ & Relapse $\mathrm{n}=12$ & No relapse $\mathrm{n}=32$ & P univariate & OR & $\mathrm{CI}(95 \%)$ \\
\hline Age, mean(SD) & $66.2(9.53)$ & $68.8(11.12)$ & 0.524 & & \\
\hline Flap & 3 & 30 & $<0.001$ & 0.022 & $0.003-0.154$ \\
\hline Muscle flap & 3 & 23 & 0.007 & 0.130 & $0.029-0.595$ \\
\hline Fat flap & 0 & 7 & 0.088 & 0.676 & $0.540-0.845$ \\
\hline Nicotine abuse & 4 & 5 & 0.188 & 2.700 & $0.583-12.511$ \\
\hline Numbness of lip & 7 & 11 & 0.137 & 2.673 & $0.686-10.412$ \\
\hline Disease malignant vs. benign & 11 & 26 & 0.372 & 2.538 & $0.273-23.638$ \\
\hline Lower vs. upper jaw & 10 & 25 & 0.531 & 1.400 & $0.247-7.930$ \\
\hline Front vs back of jaw & 5 & 3 & 0.025 & 6.905 & $1.323-36.035$ \\
\hline Max. clinical diameter of necrosis in cm (SD) & $1.35(1.22)$ & $1.21(1.39)$ & 0.725 & & $1.030-0.751$ \\
\hline Max. radiologic diameter of necrosis in $\mathrm{cm}(\mathrm{SD})$ & $2.47(0.52)$ & $2.66(0.761)$ & 0.348 & & $0.221-0.608$ \\
\hline Duration of necrosis in month (SD) & $21.0(18.2)$ & $11(14.4)$ & 0.129 & & $3.024-21.711$ \\
\hline Duration of antiresorptive therapy in month (SD) & $77.5(92.5)$ & $48.6(38.6)$ & 0.315 & & $30.989-88.801$ \\
\hline
\end{tabular}

cases developed a temporary wound insufficiency without exposed bone. At the last point of follow-up, after secondary wound healing 24 of the 26 patients treated with the muscle flap showed mucosal integrity and therefore were evaluated as a success.

For the treatment of osteonecrosis of the upper jaw we used the Buccal Fat Flap, if the necrosis was located lateral and the fat pad was big enough to pull it over the jawbone without damaging other structures. Otherwise, similar to the lower jaw, we used the mucoperiosteal flap only. The Buccal Fat Flap was mentioned first in 1977 and is proved in cases of oroantral communication (Dolanmaz et al., 2004; Egyedi, 1977; Park et al., 2019; Poeschl et al., 2009). Regarding medication-related osteonecrosis of the upper jaw, many recent studies show high success rates by using this flap. In the study by Aljohani et al., mucosal closure was reached in 12 of 14 cases by using the Buccal Fat Flap for wound closure. Thirteen of the cases exhibited an oroantral communication before surgery and are therefore related to stage III due to the AAOMS (Aljohani et al., 2019; Ruggiero et al., 2014). Ristow et al. reached a success rate of $93.1 \%$ (27/29) based on all stages (Ristow et al., 2018). Unfortunately, in our study only seven cases treated with the Buccal Fat Flap have been included.
However, the success rate of $100 \%$ regarding this group stands in line with results of earlier studies up to ten cases (Berrone et al., 2015; Gallego et al., 2012; Rotaru et al., 2015).

Regarding the different surgical procedures, it must be emphasized that, compared to the patients treated with either the muscle or the fat flap, the outcome of patients treated with the mucoperiosteal flap only ( $n=11$ ) is significantly worse. This group developed more relapses than the group treated with the muscle or the fat flap ( $\mathrm{p}<0.001)$ and only 6 patients reached mucosal integrity at the end of the study.

This confirms the results of Mücke and others. Here, a reduction of the risk of recurrence by using the Mylohyoid Muscle Flap $(n=20)$ compared to the use of the mucoperiosteal flap $(n=169)$ has also been reported (Mücke et al., 2016).

Besides a positive surgical outcome, an improvement of the quality of life is an important aspect of a successful therapy. Most of the cases of MRONJ are related to a malignant disease which itself leads to prolonged hospitalizations for the patient. In spite of the promising results of our study, one must keep in mind that many patients went through a long healing process. In cases of a 


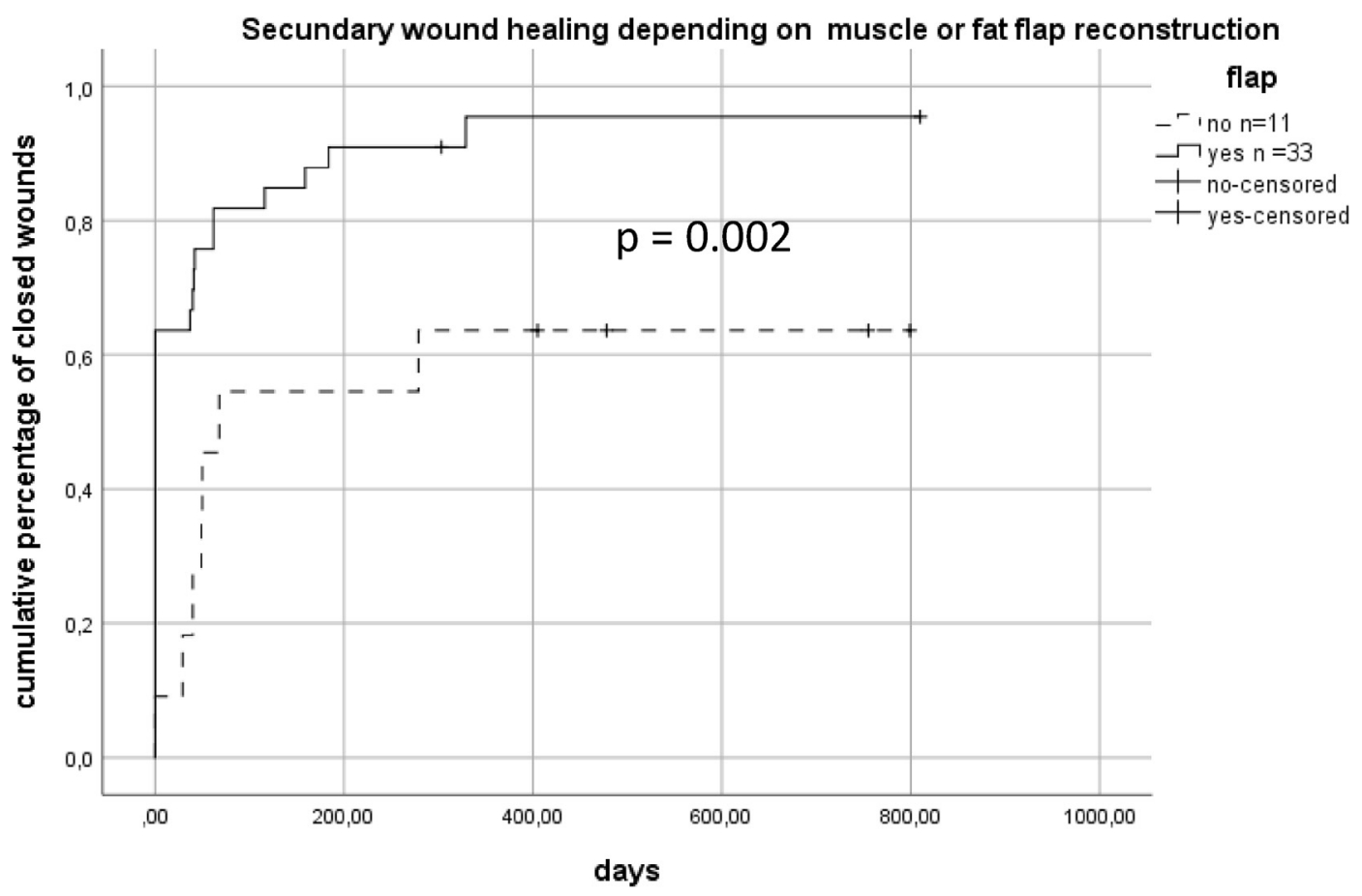

Fig. 3. Secondary wound healing depending on muscle or fat flap reconstruction.

Table 3

Side effects of MRONJ.

\begin{tabular}{|c|c|c|c|c|c|}
\hline $\mathrm{n}$ & Pre Op & Post Op & $\mathrm{p}$ & OR & $\mathrm{CI}(95 \%)$ \\
\hline Pain (scale,SD) & $3.54(3.33)$ & $0.93(2.19)$ & $<0.001$ & & \\
\hline Definite Prosthetic rehabilitation direct postop & 26 & 11 & 0.001 & 1.733 & $1.247-2.409$ \\
\hline Definite Prosthetic rehabilitation late postop & 26. & 31 & 0.213 & 0.794 & $0.520-1.214$ \\
\hline Halitosis & 19 & 0 & $<0.001$ & a & a \\
\hline Numbness lip & 0 & 18 & $<0.001$ & a & a \\
\hline Numbness tongue & 0 & 0 & a & & \\
\hline
\end{tabular}

a Statistical analysis not possible.

temporary wound insufficiency (16/44) the time until full mucosal closure was reached ranged between 29 and 329 days with a mean duration of 99.2 days. For the best result of each individual patient and an avoidance of iterations of surgery, an intensive follow-up might be necessary.

Most of the patients with advanced necrosis also suffer from pain, chewing problems and halitosis. Our results show a postoperative decrease of pain from $3.54 / 10$ to $0.93 / 10$ on average on a numerical pain rating scale and a reduction of halitosis to $0 \%$ after surgery. One may doubt the possibility of prosthetic rehabilitation after lifting up the mouth base by using the Mylohyoid Muscle Flap. We found out that pre-operatively 17 out of 26 patients had received a prosthetic service before. Postoperatively, after a temporary worsening of the prosthetic supply during the time of wound healing, long-term results showed a successful prosthetic rehabilitation in 18 of 26 of the cases. Hence, a significant improvement of the prosthetic care has not been seen, but there was no worsening either. Nevertheless, more research about long-term prosthetic rehabilitation in relation with the flap would be helpful.

Despite the promising outcome by the use of the Mylohyoid Muscle Flap and Buccal Fat Flap we see some limitations of the study. The flaps have only been used if the anatomical conditions were favorable. Especially, if the mylohyoid muscle was too thin for the preparation only a mucoperiosteal flap was possible for wound closure. Therefore, patients were not randomized referring to the different techniques. Furthermore, although all patients had a
MRONJ stage III the general state of health was variable, as most patients suffered $\mathrm{f}$ a malignant disease, which may have affected the wound healing.

\section{Conclusion}

We have shown that the Mylohyoid Muscle Flap and the Buccal Fat Flap reached better results than the mucoperiosteal flap alone and are therefore a good alternative to the usual procedure of soft tissue closure in cases of advanced MRONJ. In spite of prolonged healing processes, the oral condition has improved in many respects.

The Mylohyoid Muscle Flap and the Buccal Fat Flap are promising procedures in the treatment of patients with MRONJ. Since most of the studies show a small amount of cases particularly in the number of advanced stages on MRONJ, more research is needed.

\section{Declaration of Competing Interest}

None declared.

\section{References}

Aljohani S, Troeltzsch M, Hafner S, Kaeppler G, Mast G, Otto S: Surgical treatment of medication-related osteonecrosis of the upper jaw: case series. Oral Dis 25: 497-507, 2019

Amgen Canada Inc.: Prolia Product Monograph; 2019 
Berrone M, Florindi FU, Carbone V, Aldiano C, Pentenero M: Stage 3 medication-related osteonecrosis of the posterior maxilla: surgical treatment using a pedicled buccal fat pad flap: case reports. J Oral Maxillofac Surg 73: 2082-2086, 2015

Bodem JP, Schaal C, Kargus S, Saure D, Mertens C, Engel M, et al: Surgical management of bisphosphonate-related osteonecrosis of the jaw stages II and III. Oral Surg Oral Med Oral Pathol Oral Radiol 121: 367-372, 2016

Carlson ER: Management of antiresorptive osteonecrosis of the jaws with primary surgical resection. J Oral Maxillofac Surg 72: 655-657, 2014

Carlson ER, Basile JD: The role of surgical resection in the management of bisphosphonate-related osteonecrosis of the jaws. J Oral Maxillofac Surg 67: 85-95, 2009

Coleman R, Woodward E, Brown J, Cameron D, Bell R, Dodwell D, et al: Safety of zoledronic acid and incidence of osteonecrosis of the jaw (ONJ) during adjuvant therapy in a randomised phase III trial (AZURE: BIG 01-04) for women with stage II/III breast cancer. Breast Cancer Res Treat 127: 429-438, 2011

Dolanmaz D, Tuz H, Bayraktar S, Metin M, Erdem E, Baykul T: Use of pedicled bucca fat pad in the closure of oroantral communication: analysis of 75 cases. Quintessence Int 35: 241-246, 2004

Eckardt A, Lemound J, Lindhorst D, Rana M, Gellrich N-C: Surgical management of bisphosphonate-related osteonecrosis of the jaw in oncologic patients: a challanging problem. Anticancer Res 31: 2313-2318, 2011

Egyedi P: Utilization of the buccal fat pad for closure of oro-antral and/or oro-nasal communications. J Maxillofac Surg 5: 241-244, 1977

Gallego L, Junquera L, Pelaz A, Hernando J, Megías J: The use of pedicled buccal fat pad combined with sequestrectomy in bisphosphonate-related osteonecrosis of the maxilla. Med Oral Patol Oral Cir Bucal 17: e236-e241, 2012

Graziani F, Vescovi P, Campisi G, Favia G, Gabriele M, Gaeta GM, et al: Resective surgical approach shows a high performance in the management of advanced cases of bisphosphonate-related osteonecrosis of the jaws: a retrospective survey of 347 cases. J Oral Maxillofac Surg 70: 2501-2507, 2012

Hallmer F, Andersson G, Gotrick B, Warfvinge G, Anderud J, Bjornland T: Prevalence, initiating factor, and treatment outcome of medication-related osteonecrosis of the jaw-a 4-year prospective study. Oral Surg Oral Med Oral Pathol Oral Radiol 126: 477-485, 2018

Hoff AO, Toth BB, Altundag K, Johnson MM, Warneke CL, Hu M, et al: Frequency and risk factors associated with osteonecrosis of the jaw in cancer patients treated with intravenous bisphosphonates. J Bone Miner Res 23: 826-836, 2008

Klingelhöffer C, Zeman F, Meier J, Reichert TE, Ettl T: Evaluation of surgical outcome and influencing risk factors in patients with medication-related osteonecrosis of the jaws. J Craniomaxillofac Surg 44: 1694-1699, 2016

Lee S-H, Chang S-S, Lee M, Chan R-C, Lee C-C: Risk of osteonecrosis in patients taking bisphosphonates for prevention of osteoporosis: a systematic review and meta-analysis. Osteoporos Int 25: 1131-1139, 2014

Lemound J, Eckardt A, Kokemüller H, See Cvon, Voss PJ, Tavassol F, et al: Bisphosphonate-associated osteonecrosis of the mandible: reliable soft tissue reconstruction using a local myofascial flap. Clin Oral Investig 16: 1143-1152, 2012

Lo JC, O'Ryan FS, Gordon NP, Yang J, Hui RL, Martin D, et al: Prevalence of osteonecrosis of the jaw in patients with oral bisphosphonate exposure. J Ora Maxillofac Surg 68: 243-253, 2010

Marx RE: Pamidronate (Aredia) and zoledronate (Zometa) induced avascular necrosis of the jaws. J Oral Maxillofac Surg 61: 1115-1117, 2003

Marx RE, Sawatari Y, Fortin M, Broumand V: Bisphosphonate-induced exposed bone (osteonecrosis/osteopetrosis) of the jaws: risk factors, recognition, prevention, and treatment. J Oral Maxillofac Surg 63: 1567-1575, 2005

Mauri D, Valachis A, Polyzos IP, Polyzos NP, Kamposioras K, Pesce LL: Osteonecrosis of the jaw and use of bisphosphonates in adjuvant breast cancer treatment: a meta-analysis. Breast Cancer Res Treat 116: 433-439, 2009
Mücke T, Koerdt S, Jung M, Mitchell DA, Wolff K-D, Kesting MR, et al: The role of mylohyoid flap in the treatment of bisphosphonate-related osteonecrosis of the jaws. J Craniomaxillofac Surg 44: 369-373, 2016

Otto S, Schreyer C, Hafner S, Mast G, Ehrenfeld M, Stürzenbaum S, et al: Bisphosphonate-related osteonecrosis of the jaws - characteristics, risk factors, clinical features, localization and impact on oncological treatment. J Craniomaxillofac Surg 40: 303-339, 2012

Park J, Chun B-d, Kim U-K, Choi N-R, Choi H-S, Hwang D-S: Versatility of the pedicled buccal fat pad flap for the management of oroantral fistula: a retrospective study of 25 cases. Maxillofac Plast Reconstr Surg 41: 50, 2019

Poeschl PW, Baumann A, Russmueller G, Poeschl E, Klug C, Ewers R: Closure of oroantral communications with Bichat's buccal fat pad. J Oral Maxillofac Surg 67: 1460-1466, 2009

Qi W-X, Tang L-N, He A-N, Yao Y, Shen Z: Risk of osteonecrosis of the jaw in cancer patients receiving denosumab: a meta-analysis of seven randomized controlled trials. Int J Clin Oncol 19: 403-410, 2014

Reich W, Bilkenroth U, Schubert J, Wickenhauser C, Eckert AW: Surgical treatment of bisphosphonate-associated osteonecrosis: prognostic score and long-term results. J Craniomaxillofac Surg 43: 1809-1822, 2015

Ristow O, Otto S, Troeltzsch M, Hohlweg-Majert B, Pautke C: Treatment perspectives for medication-related osteonecrosis of the jaw (MRONJ). J Craniomaxillofac Surg 43: 290-293, 2015

Ristow O, Rückschloß T, Bodem J, Berger M, Bodem E, Kargus S, et al: Double-layer closure techniques after bone surgery of medication-related osteonecrosis of the jaw - a single center cohort study. J Craniomaxillofac Surg 46: 815-824, 2018

Rotaru H, Kim M-K, Kim S-G, Park Y-W: Pedicled buccal fat pad flap as a reliable surgical strategy for the treatment of medication-related osteonecrosis of the jaw. J Oral Maxillofac Surg 73: 437-442, 2015

Rugani P, Walter C, Kirnbauer B, Acham S, Begus-Nahrman Y, Jakse N: Prevalence of medication-related osteonecrosis of the jaw in patients with breast cancer, prostate cancer, and multiple myeloma. Dent J (Basel) 4, 2016

Ruggiero SL, Dodson TB, Assael LA, Landesberg R, Marx RE, Mehrotra B: American Association of Oral and Maxillofacial Surgeons position paper on bisphosphonate-related osteonecrosis of the jaws-2009 update. J Oral Maxillofac Surg 67: 2-12, 2009

Ruggiero SL, Dodson TB, Fantasia J, Goodday R, Aghaloo T, Mehrotra B, et al: American Association of Oral and Maxillofacial Surgeons position paper on medication-related osteonecrosis of the jaw-2014 update. J Oral Maxillofac Surg 72: 1938-1956, 2014

Russell RGG, Watts NB, Ebetino FH, Rogers MJ: Mechanisms of action of bisphosphonates: similarities and differences and their potential influence on clinical efficacy. Osteoporos Int 19: 733-759, 2008

Saussez S, Javadian R, Hupin C, Magremanne M, Chantrain G, Loeb I, et al: Bisphosphonate-related osteonecrosis of the jaw and its associated risk factors: a Belgian case series. Laryngoscope 323(9 119), 2009

Vahtsevanos K, Kyrgidis A, Verrou E, Katodritou E, Triaridis S, Andreadis CG, et al: Longitudinal cohort study of risk factors in cancer patients of bisphosphonate-related osteonecrosis of the jaw. J Clin Oncol 27: 5356-5362, 2009

Wilde F, Heufelder M, Winter K, Hendricks J, Frerich B, Schramm A, et al: The role of surgical therapy in the management of intravenous bisphosphonates-related osteonecrosis of the jaw. Oral Surg Oral Med Oral Pathol Oral Radiol Endod 111: 153-163, 2011

Wutzl A, Biedermann E, Wanschitz F, Seemann R, Klug C, Baumann A, et al: Treatment results of bisphosphonate-related osteonecrosis of the jaws. Head Neck 30: 1224-1230, 2008 